\title{
Risk Factors for Disease Behavior Evolution and Efficacy of Biologics in Reducing Progression in Pediatric Patients with Nonstricturing, Nonpenetrating Crohn's Disease at Diagnosis: A Single-Center Experience in Korea
}

\author{
Hyun Jin Kim¹, Seak Hee Oh², Sung Hee Lee², Yu-Bin Kim², Dae Yeon Kim³, Sang Hyoung Park , Byong Duk Ye4, \\ Suk-Kyun Yang ${ }^{4}$, and Kyung Mo Kim² \\ ${ }^{1}$ Department of Pediatrics, Chungnam National University Hospital, Daejeon, Departments of ${ }^{2}$ Pediatrics and ${ }^{3}$ Pediatric Surgery, Asan \\ Medical Center Children's Hospital, University of Ulsan College of Medicine, and ${ }^{4}$ Department of Gastroenterology, Asan Medical \\ Center, University of Ulsan College of Medicine, Seoul, Korea
}

See editorial on page 791.

\section{Article Info}

Received September 2, 2020

Revised December 15, 2020

Accepted December 15, 2020

\section{Corresponding Author}

\section{Kyung Mo Kim}

ORCID https://orcid.org/0000-0001-7896-6751

E-mailkmkim@amc.seoul.kr

\begin{abstract}
Background/Aims: Recently, the treatment of Crohn's disease (CD) has changed to a treat-totarget strategy, in which disease progression is prevented with early intervention. We analyzed the long-term evolution of nonstricturing, nonpenetrating (B1) disease at diagnosis and factors related to disease evolution in pediatric $C D$.

Methods: We retrospectively analyzed 402 patients between 2000 and 2013 who were younger than 18 years and had B1 disease at $\mathrm{CD}$ diagnosis. The median follow-up was 6.1 years (range, 1 to 13 years). The cumulative probabilities of developing stricturing (B2) or penetrating (B3) disease and associations between risk factors and disease behavior evolution were evaluated.

Results: Among the 402 patients, 75 (18.7\%) had B2 or B3 disease by the final follow-up. The cumulative probabilities of disease behavior evolution were $18.3 \%, 34.3 \%$, and $50.9 \%$ at 5,10 , and 13 years, respectively. Patients whose disease progressed had an increased risk of intestinal resection (hazard ratio [HR], 3.61; 95\% confidence interval [Cl], 2.25 to $6.03 ; p<0.001$ ). Firstdegree family history of inflammatory bowel disease (HR, 2.38; $95 \% \mathrm{Cl}, 1.07$ to $5.28 ; p=0.032$ ), isolated ileal involvement at diagnosis ( $\mathrm{HR}, 7.55 ; 95 \% \mathrm{Cl}, 1.04$ to $15.57 ; \mathrm{p}=0.045)$, and positive anti-Saccharomyces cerevisiae antibody titers (HR, 2.10;95\% Cl, 1.03 to $4.25 ; p=0.040$ ) were associated with disease behavior evolution. Early treatment with biologics significantly reduced disease progression (HR, $0.46 ; 95 \% \mathrm{Cl}, 0.79$ to 3.39 ; $\mathrm{p}=0.042$ ).

Conclusions: This study suggests that early aggressive therapy should be considered in B1 behavior pediatric $C D$ patients with risk factors of disease evolution to improve long-term outcomes. (Gut Liver 2021;15:851-857)
\end{abstract}

Key Words: Pediatrics; Crohn disease; Behavior; Evolution

\section{INTRODUCTION}

Crohn's disease (CD) is a chronic progressive and disabling disease. Inflammation in the intestinal tract causes symptoms such as diarrhea and abdominal pain and leads to irreversible damages such as strictures and fistulas which can result in hospitalizations and intestinal surgeries. ${ }^{1}$

An adult study on the disease evolution of $\mathrm{CD}$ reported that $80 \%$ of patients ultimately showed a change to a stricturing (B2) or penetrating (B3) disease behavior. ${ }^{2}$ In 594 Asian children, we reported that $>80 \%$ showed disease evaluation into a B2 or B3 behavior at 20 years of followup. ${ }^{3}$ In a study on Western children, $60 \%$ showed disease evolution into a B2 or B3 behavior at 15 years of followup. ${ }^{4}$

Recently, the treatment of $\mathrm{CD}$ has changed to a treat-totarget strategy, in which disease progression is prevented 
with early intervention. ${ }^{5,6}$ A Western pediatric study reported that early use of biologics delayed the progression to stricturing and/or penetrating disease; however, prevention of early surgery was not achieved with biologics.

For these reasons, treatment strategies should focus on altering the natural disease course by preventing irreversible damages. Moreover, it is more advisable to focus on patients who show a nonstricturing and nonpenetrating (B1) disease behavior at diagnosis, but are likely to develop structuring and/or penetrating behavior in the course of management. ${ }^{8}$ However, studies about Asian children are rare and there are debates about the efficacy of biologics.

Therefore, we aimed to analyze the evolution of disease behavior in $\mathrm{B} 1 \mathrm{CD}$ at diagnosis and the factors associated with disease evolution in a Korean pediatric population. We also analyzed the effect of treatment on disease behavior evolution.

\section{MATERIALS AND METHODS}

\section{Patients}

This retrospective analysis was approved by the Institutional Review Board of Asan Medical Center and was conducted in accordance with the Declaration of Helsinki (IRB number: 2014-1064). This study was a retrospective study and informed consent was waived.

We retrospectively analyzed patients who were diagnosed with $\mathrm{CD}$ before 18 years of age and were registered at the inflammatory bowel disease (IBD) center and Asan Medical Center Children's Hospital between March 2000 and December 2013. Among 450 patients, 402 (89.3\%) who had nonstricturing, nonpenetrating disease at diagnosis and available information about disease behavioral evolutions over at least 1 year of follow-up were enrolled. Patients with monogenic IBD, such as those with interleukin-10 receptor mutation and X-linked inhibitor of apoptosis deficiency, were excluded from this study. ${ }^{9}$

The diagnosis of $\mathrm{CD}$ was based on conventional clinical, endoscopic, radiologic, and histologic criteria. Patients with unclassified IBD, infections, or other recognized causes of intestinal inflammation were excluded. ${ }^{10}$

\section{Study design}

All data were collected from the IBD registry of Asan Medical Center or obtained through a retrospective review of medical records.

The baseline demographic and clinical characteristics, including sex, age at diagnosis, first-degree family history of IBD, disease behavior and location, presence of perianal fistula, and growth delay, were evaluated. The titers of
anti-Saccharomyces cerevisiae (ASCA) immunoglobulin $\mathrm{G}(\mathrm{IgG}) / \operatorname{IgA}$ were measured at the time of diagnosis. Age at diagnosis, disease location and behavior of $\mathrm{CD}$ were categorized according to the Paris classification. ${ }^{11}$ Disease behavior was classified as B1 (nonstricturing, nonpenetrating), B2 (stricturing), or B3 (penetrating). Growth delay was defined using the height $\mathrm{z}$-score at diagnosis or when the subsequent height $\mathrm{z}$-score was significantly less than expected.

Our treatment policy is to step up the therapy when the patients become unresponsive to first-line agents. ${ }^{12}$ Thiopurines were used as maintenance treatment after remission and in case of biologics, we used them who showed no response to first treatment such as exclusive enteral nutrition and steroid or had perianal lesion. We use exclusive enteral nutrition as the first-line treatment more frequently than steroids. ${ }^{13,14}$ Early use of thiopurines or anti-tumor necrosis factor agents was defined as treatment starting within 3 months of diagnosis and before the occurrence of complications (intestinal surgery or disease behavior evolution).

\section{Statistical analysis}

Discrete data are expressed as numbers and percentages. We used chi-square tests or Fisher exact tests to compare binominal variables. The cumulative probabilities of disease behavior evolution were calculated using the KaplanMeier method. To quantify risks, we used the hazard ratios (HRs) of the Cox proportional hazard models. All prognostic variables with $\mathrm{p}<0.1$ according to the univariate analysis were included in the multivariate analysis. A value of $\mathrm{p}<0.05$ was considered statistically significant. Statistical analysis was performed using SPSS version 18.0 (IBM Corp., Armonk, NY, USA).

\section{RESULTS}

\section{Baseline characteristics}

Of the 402 patients included in the present study, 298 (74.1\%) were males and 104 (25.9\%) were females, the median age at diagnosis and duration of follow-up were 15 years (range, 2 to 17 years) and 6.1 years (range, 1 to 13 years), respectively. Perianal fistula was observed at diagnosis in 202 patients (50.2\%) and a positive first-degree family history of IBD was recorded in 23 patients (5.7\%). For treatment, thiopurines and anti-tumor necrosis factor agents were used in $71.4 \%$ (287/402) and 20.9\% (84/402) of patients, respectively. The baseline characteristics at diagnosis of the 402 patients are summarized in Table 1. 


\section{Evolution of disease behavior}

Of the 402 patients with $\mathrm{B} 1$ disease at diagnosis, 75 (18.7\%) progressed to B2 and/or B3 disease by the final follow-up. The cumulative probabilities of disease behavior evolution (either stricturing or penetrating disease) were $1.3 \%$ at 1 year, $18.3 \%$ at 5 years, $34.3 \%$ at 10 years, and

Table 1. Baseline Characteristics of 402 Pediatric Patients with Nonstricturing, Nonpenetrating Crohn's Disease at Diagnosis

\begin{tabular}{lc}
\hline \multicolumn{1}{c}{ Characteristics } & No. $(\%)$ \\
\hline Male sex & $298(74.1)$ \\
Age at diagnosis, yr & $10(2.5)$ \\
A1a & $308(76.6)$ \\
A1b & $84(20.9)$ \\
A2 & \\
Location at diagnosis & $42(10.5)$ \\
L1 & $32(8.0)$ \\
L2 & $324(80.8)$ \\
L3 & $3(0.7)$ \\
L4 only & $85(21.1)$ \\
Upper disease at diagnosis & $3(3.5)$ \\
L4 only & $29(34.1)$ \\
+L4a & $53(62.4)$ \\
+L4b & $202(50.2)$ \\
Perianal fistula at diagnosis & \\
Growth impairment & $370(92.0)$ \\
G0 & $32(8.0)$ \\
G1 & $23(5.7)$ \\
First-degree family history of IBD & $199(74.5)$ \\
Positive ASCA (n=267) & \\
Use of medication & $287(71.4)$ \\
Thiopurines & $84(20.9)$ \\
Anti-TNF-agents & \\
\hline
\end{tabular}

L4a/L4b may coexist with L1, L2, or L3. Age at diagnosis, disease location, behavior, and growth impairment were categorized according to the Paris classification.

IBD, inflammatory bowel disease; ASCA, anti-Saccharomyces cerevisiae antibodies; TNF, tumor necrosis factor.

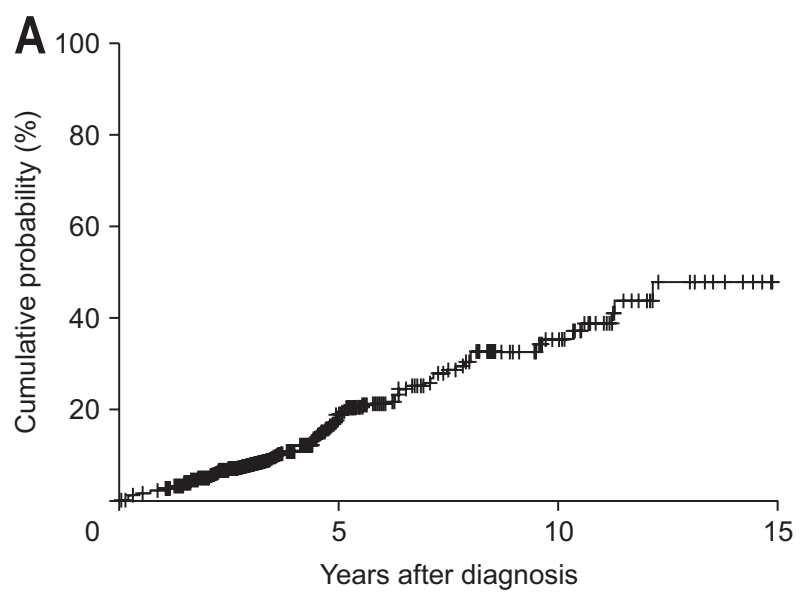

$50.9 \%$ at 13 years (Fig. 1A). The cumulative probabilities of stricturing disease were $1.0 \%$ at 1 year, $17.7 \%$ at 5 years, $32.4 \%$ at 10 years, and $49.4 \%$ at 13 years. The cumulative probabilities of penetrating disease were $0.5 \%$ at 1 year, $5.9 \%$ at 5 years, $14.9 \%$ at 10 years, and $36.2 \%$ at 13 years.

\section{Association of disease behavior evolution and intestinal resection}

Patients who showed evolution of disease behavior had a higher risk of intestinal resection than those whose disease behavior remained as $\mathrm{B} 1$ ( $\mathrm{HR}, 3.61 ; 95 \%$ confidence interval [CI], 2.25 to 6.03 ; $\mathrm{p}<0.001$ ) (Fig. 2).

\section{Association of disease behavior evolution and related factors at diagnosis}

In multivariate Cox analysis, patients with isolated ileal involvement at diagnosis had a higher risk of disease behavior evolution than those with colon involvement (L2 and L3) (HR, 7.55; 95\% CI, 1.04 to 15.57; $\mathrm{p}=0.045$ ). Moreover, patients with a first-degree family history of IBD (HR, 2.38; 95\% CI, 1.07 to 5.28; $\mathrm{p}=0.032$ ) and positive ASCA IgG and/or IgA titers (HR, 2.10; 95\% CI, 1.03 to 4.25; $\mathrm{p}=0.040$ ) had a higher risk of disease behavior evolution than those without such characteristics. The factors associated with the evolution of disease behavior are summarized in Table 2.

\section{Association of disease behavior evolution and treatment}

In multivariate Cox analysis, patients with early treatment with biologics had a lower risk of disease behavior evolution than those without (HR, 0.46; 95\% CI, 0.79 to 3.39; $\mathrm{p}=0.042$ ) (Fig. 1B). Early thiopurine treatment had no effect on the evolution of disease behavior (HR, 0.93; 95\% CI, 0.61 to $1.42 ; \mathrm{p}=0.753$ ).

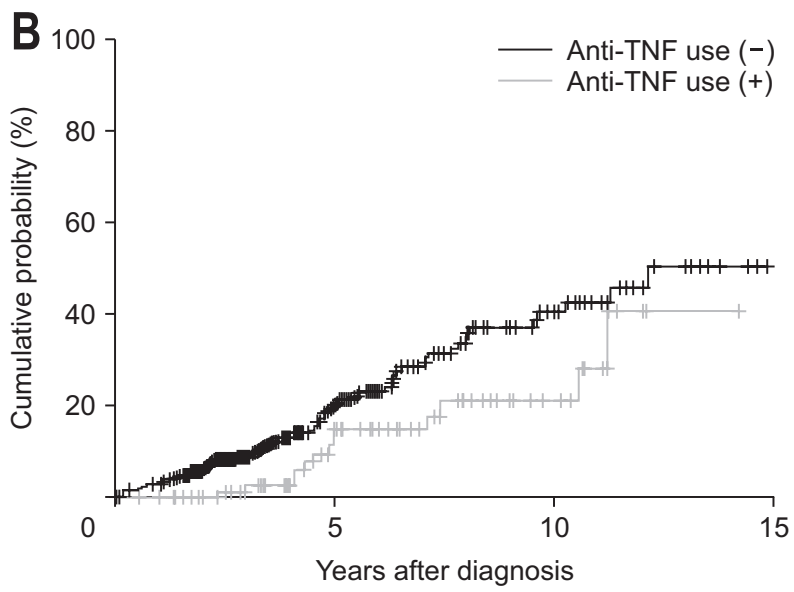

Fig. 1. Cumulative probability of the evolution of disease behavior in Crohn's disease patients with B1 behavior at diagnosis (A) and according to use of anti-tumor necrosis factor (TNF; $p=0.042$ ) (B). 


\section{DISCUSSION}

Our study showed the long-term course of disease behavior evolution in $\mathrm{CD}$, with cumulative probabilities of $34.3 \%$ at 10 years and $50.9 \%$ at 13 years. We focused on pediatric patients who had nonstricturing, nonpenetrating behavior at diagnosis and found several related factors with behavior evolution. Furthermore, the possibility of

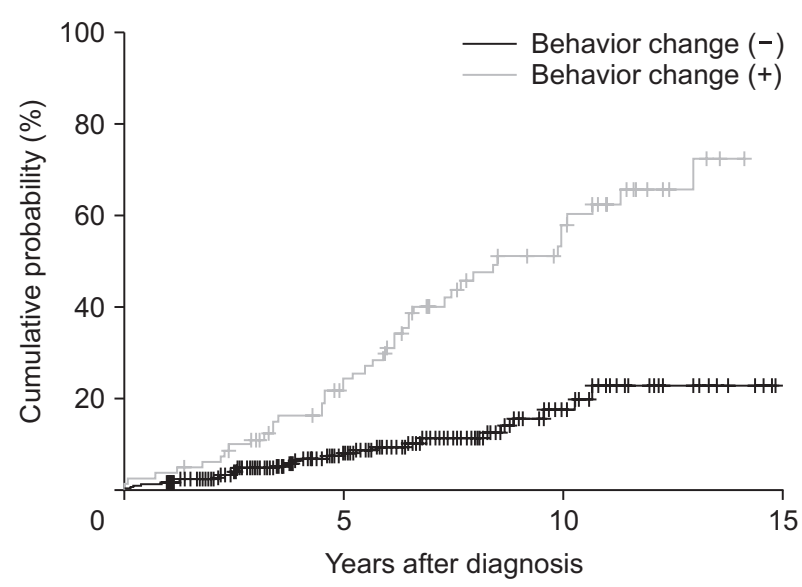

Fig. 2. Cumulative probability of intestinal resection in Crohn's disease patients according to the evolution of disease behavior $(p<0.001)$. early biologics in reducing progression was suggested. These findings support the recent treat-to-target approach for $\mathrm{CD}$ management, even in pediatric patients.

A recent study reported that a change in disease location and the progression of disease behavior were significant risk factors for an increased cumulative risk of intestinal resection, similar to the findings of our study, and suggested the possible role of biologics in reducing changes in disease location. ${ }^{15}$

As mentioned earlier, several factors are associated with disease evolution. First, disease location is a significant predictive factor for the evolution of disease behavior. Both in adults and children, ileal involvement has been considered a significant predictor of disease behavior evolution. ${ }^{16,17}$ In our study, patients with isolated ileal involvement at diagnosis had an increased risk of disease behavior evolution, relative to those with colonic involvement (HR, 7.55; 95\% CI, 1.04 to 15.57; $\mathrm{p}=0.045)$. Impaired autophagy which results in reduced bacterial clearance and different microbial composition might be the cause of complicated course of ileal involvement. ${ }^{18}$

Second, interestingly, evolution of disease behavior significantly more often occurred in patients with a firstdegree family history of IBD (HR, 2.38; 95\% CI, 1.07 to 5.28; $\mathrm{p}=0.032$ ). A previous Korean study showed that fam-

Table 2. Univariate and Multivariate Cox Analyses of Risk Factors for Disease Behavior Evolution in Pediatric Patients with Nonstricturing, Nonpenetrating Crohn's Disease at Diagnosis

\begin{tabular}{|c|c|c|c|c|}
\hline \multirow{2}{*}{ Factor } & \multicolumn{2}{|c|}{ Univariate analysis } & \multicolumn{2}{|c|}{ Multivariate analysis } \\
\hline & $\mathrm{HR}(95 \% \mathrm{Cl})$ & p-value & $\mathrm{HR}(95 \% \mathrm{Cl})$ & $\mathrm{p}$-value \\
\hline \multicolumn{5}{|l|}{ Sex } \\
\hline Male & Reference & & & \\
\hline Female & $1.41(0.86-2.29)$ & 0.163 & & \\
\hline \multicolumn{5}{|l|}{ Family history of IBD } \\
\hline No & Reference & & Reference & \\
\hline Yes & $2.72(1.23-5.97)$ & 0.013 & $2.38(1.07-5.28)$ & 0.032 \\
\hline \multicolumn{5}{|l|}{ Age at diagnosis } \\
\hline 1-9 Years & Reference & & & \\
\hline 10-17 Years & $1.10(0.27-4.52)$ & 0.887 & & \\
\hline \multicolumn{5}{|c|}{ Isolated ileal involvement at diagnosis } \\
\hline No & Reference & & Reference & \\
\hline Yes & $8.72(1.21-18.04)$ & 0.031 & $7.55(1.04-15.57)$ & 0.045 \\
\hline \multicolumn{5}{|l|}{ Perianal fistula at diagnosis } \\
\hline No & Reference & & & \\
\hline Yes & $0.82(0.52-1.31)$ & 0.422 & & \\
\hline \multicolumn{5}{|l|}{ Growth impairment } \\
\hline No & Reference & & & \\
\hline Yes & $1.14(0.54-2.38)$ & 0.720 & & \\
\hline Positive ASCA & $2.03(1.01-4.12)$ & 0.048 & $2.10(1.03-4.25)$ & 0.040 \\
\hline \multicolumn{5}{|l|}{ Early use of medication } \\
\hline Thiopurines & $0.93(0.61-1.42)$ & 0.753 & & \\
\hline Anti-TNF agents & $0.48(0.86-3.06)$ & 0.046 & $0.46(0.79-3.39)$ & 0.042 \\
\hline
\end{tabular}

Age at diagnosis, disease location, behavior, and growth impairment were categorized according to the Paris classification.

$\mathrm{HR}$, hazard ratio; $\mathrm{Cl}$, confidence interval; IBD, inflammatory bowel disease; ASCA, anti-Saccharomyces cerevisiae antibodies; TNF, tumor necrosis factor. 
ily history was an independent risk factor for the time to first intestinal resection in patients with CD (HR, 1.61; $95 \% \mathrm{CI}, 1.13$ to $2.29 ; \mathrm{p}=0.009$ ), suggesting the possibility of a more aggressive clinical course of $\mathrm{CD}$ similar to that in our study. ${ }^{19}$

Third, immune responses to a number of microbial antigens have been associated with complicated disease behaviors and serum immune responses predicted a more rapid progression from uncomplicated to complicated disease. ${ }^{20}$ In a recent study, a positive ASCA status was associated with complicated disease behaviors and increased cumulative probability of disease-related surgery. ${ }^{16} \mathrm{~A}$ pediatric prospective study also showed a significant association between the development of a complicated CD behavior and the presence of antibodies during a median followup of 18 months. ${ }^{21} \mathrm{~A}$ meta-analysis conducted by Zhang et al. ${ }^{22}$ noted that ASCA was a risk factor for early-onset disease, ileal involvement, complicated disease behavior, perianal disease, and the need for surgery for CD. In our study, the presence of ASCA IgG/IgA significantly increased the risk of disease behavior evolution in patients with an uncomplicated disease behavior at diagnosis (HR, 2.10; 95\% CI, 1.03 to $4.25 ; \mathrm{p}=0.040$ ). Also, complicated behavior was more commonly observed in patients with ASCA IgG/IgA ( $10.8 \%$ vs $3.6 \%, p=0.029$, data not shown). However, in our study perianal CD and small bowel involvement was not associated with that.

The presence of perianal disease has been considered a poor prognostic factor for $\mathrm{CD}$ and early aggressive treatment that alters the natural history of the disease is recommended. ${ }^{23,24}$ Two other studies also indicated that perianal disease was a significant predictor of disease behavior evolution. ${ }^{25,26}$ Kaur et al. ${ }^{27}$ found that patients with perianal $\mathrm{CD}$ demonstrated a higher prevalence of antibodies and higher median levels of antibodies (ASCA IgG, ASCA IgA, I2, Omp C, and C-Bir), which are typically associated with complicated small bowel involvement in $\mathrm{CD}$, suggesting that distinct immune processes may contribute to the pathogenesis of complicated disease in small bowel and perianal pathologies. However, in our study, perianal fistula at diagnosis did not predict the evolution of disease behavior. Previously, we reported a higher prevalence of perianal fistula in Koreans than that reported in Western studies, as well as the lack of association between the cumulative probabilities of intestinal resection and perianal fistula at diagnosis. ${ }^{3,28}$ Larger prospective studies should be performed to resolve these discrepancies.

Biologics have the potential of achieving mucosal healing and can prevent stricturing and/or penetrating com- plications..$^{29}$ Although relevant data are relatively scarce in children, studies on children also reported that biologics achieved short-term mucosal healing, ${ }^{30,31}$ which can translate to reduced hospitalizations and surgeries in the longterm. ${ }^{32}$ Recent studies reported that early use of biologics delayed disease progression, ${ }^{7,33}$ and the overall clinical and growth outcomes were also better in groups treated with early biologics. ${ }^{34}$ In this study, the cumulative probability of disease behavior evolution was decreased by the early use of biologics. In these groups, after 10 years with disease, the probability of disease behavior evolution was $27.4 \%$. However, thiopurines were not effective in reducing disease progression. As opinions about the role of immunosuppressants are conflicting, more studies may be needed in the future. 35,36

The European Crohn's and Colitis Organization and the European Society for Paediatric Gastroenterology Hepatology and Nutrition guidelines suggest early and aggressive treatment for selected patients with deep colonic ulcerations, persistent severe disease despite adequate induction therapy, growth delay, severe osteoporosis, stricturing and penetrating disease at diagnosis, and severe perianal disease. ${ }^{24}$ These conditions were based on an adult study, and complicated disease at diagnosis may be less responsive to early aggressive treatment. Patients with isolated ileal involvement at diagnosis, a first-degree family history of IBD, and positive ASCA titers had an increased risk of disease behavior progression in our study. Early biologic treatment was effective in reducing such progression. Therefore, patients with such characteristics should be paid more attention and started on treatment early.

The main limitation of our study was the retrospective design. We retrospectively applied the Paris classification according to previous data. However, we included only patients who had complete information for classification and started treatment before the occurrence of complications. We could not evaluate the effect of biologics in combination with immunosuppressive agents owing to insufficient data. Genetic factors that influence disease behavior evolution and treatment response were, also, not considered in this study.

In our analysis of the long-term course of nonstricturing, nonpenetrating $\mathrm{CD}$ in a Korean pediatric population, patients who had isolated ileal involvement at diagnosis, a first-degree family history of IBD, and positive ASCA titers showed a higher risk of disease behavior progression. Identifying relevant risk factors for the progression of disease behavior and providing early adequate treatment to prevent such progression are important. 


\section{CONFLICTS OF INTEREST}

No potential conflict of interest relevant to this article was reported.

\section{AUTHOR CONTRIBUTIONS}

Conception and design of the study: H.J.K., S.K.Y., K.M.K. Acquisition, analysis, and interpretation of data: H.J.K., S.H.O., S.H.L., Y.B.K., D.Y.K., S.H.P., B.D.Y., S.K.Y., K.M.K. Drafting of the initial manuscript: H.J.K. Critical revision for important intellectual content: H.J.K., K.M.K.

\section{ORCID}

Hyun Jin Kim

https://orcid.org/0000-0003-0279-7925

Seak Hee Oh

Sung Hee Lee

Yu-Bin Kim

https://orcid.org/0000-0002-9672-8877

https://orcid.org/0000-0003-3262-9658

https://orcid.org/0000-0001-6325-6191

Dae Yeon Kim https://orcid.org/0000-0001-8852-6389

Sang Hyoung Park

https://orcid.org/0000-0002-5366-5749

Byong Duk Ye

Suk-Kyun Yang

Kyung Mo Kim

https://orcid.org/0000-0001-6647-6325

https://orcid.org/0000-0003-2772-2575

https://orcid.org/0000-0001-7896-6751

\section{REFERENCES}

1. Roda G, Chien Ng S, Kotze PG, et al. Crohn's disease. Nat Rev Dis Primers 2020;6:22.

2. Pariente B, Cosnes J, Danese S, et al. Development of the Crohn's disease digestive damage score, the Lémann score. Inflamm Bowel Dis 2011;17:1415-1422.

3. Kim HJ, Oh SH, Kim DY, et al. Clinical characteristics and long-term outcomes of paediatric Crohn's disease: a singlecentre experience. J Crohns Colitis 2017;11:157-164.

4. Fumery M, Pariente B, Sarter H, et al. Long-term outcome of pediatric-onset Crohn's disease: a population-based cohort study. Dig Liver Dis 2019;51:496-502.

5. Colombel JF, D’haens G, Lee WJ, Petersson J, Panaccione R. Outcomes and strategies to support a treat-to-target approach in inflammatory bowel disease: a systematic review. J Crohns Colitis 2020;14:254-266.

6. Moon JS. Clinical aspects and treatments for pediatric inflammatory bowel disease. Intest Res 2019;17:17-23.

7. Kerur B, Machan JT, Shapiro JM, et al. Biologics delay progression of Crohn's disease, but not early surgery, in children. Clin Gastroenterol Hepatol 2018;16:1467-1473.
8. Allen PB, Peyrin-Biroulet L. Moving towards disease modification in inflammatory bowel disease therapy. Curr Opin Gastroenterol 2013;29:397-404.

9. Shim JO. Recent advance in very early onset inflammatory bowel disease. Pediatr Gastroenterol Hepatol Nutr 2019;22:41-49.

10. Levine A, Koletzko S, Turner D, et al. ESPGHAN revised porto criteria for the diagnosis of inflammatory bowel disease in children and adolescents. J Pediatr Gastroenterol Nutr 2014;58:795-806.

11. Levine A, Griffiths A, Markowitz J, et al. Pediatric modification of the Montreal classification for inflammatory bowel disease: the Paris classification. Inflamm Bowel Dis 2011;17:1314-1321.

12. Hanauer SB. Crohn's disease: step up or top down therapy. Best Pract Res Clin Gastroenterol 2003;17:131-137.

13. Kim HJ, Kim Y, Cho JM, Oh SH, Kim KM. Therapeutic efficacy of oral enteral nutrition in pediatric Crohn's disease: a single center non-comparative retrospective study. Yonsei Med J 2016;57:1185-1191.

14. Adamji M, Day AS. An overview of the role of exclusive enteral nutrition for complicated Crohn's disease. Intest Res 2019;17:171-176.

15. Lo B, Vester-Andersen MK, Vind I, et al. Changes in disease behaviour and location in patients with Crohn's disease after seven years of follow-up: a Danish population-based inception cohort. J Crohns Colitis 2018;12:265-272.

16. Kugathasan S, Denson LA, Walters TD, et al. Prediction of complicated disease course for children newly diagnosed with Crohn's disease: a multicentre inception cohort study. Lancet 2017;389:1710-1718.

17. Lovasz BD, Lakatos L, Horvath A, et al. Evolution of disease phenotype in adult and pediatric onset Crohn's disease in a population-based cohort. World J Gastroenterol 2013;19:2217-2226.

18. Dulai PS, Singh S, Vande Casteele N, et al. Should we divide Crohn's disease into ileum-dominant and isolated colonic diseases? Clin Gastroenterol Hepatol 2019;17:2634-2643.

19. Hwang SW, Kwak MS, Kim WS, et al. Influence of a positive family history on the clinical course of inflammatory bowel disease. J Crohns Colitis 2016;10:1024-1032.

20. Dubinsky MC, Lin YC, Dutridge D, et al. Serum immune responses predict rapid disease progression among children with Crohn's disease: immune responses predict disease progression. Am J Gastroenterol 2006;101:360-367.

21. Dubinsky MC, Kugathasan S, Mei L, et al. Increased immune reactivity predicts aggressive complicating Crohn's disease in children. Clin Gastroenterol Hepatol 2008;6:1105-1111.

22. Zhang Z, Li C, Zhao X, et al. Anti-Saccharomyces cerevisiae antibodies associate with phenotypes and higher risk for surgery in Crohn's disease: a meta-analysis. Dig Dis Sci 
2012;57:2944-2954.

23. Sachar DB, Bodian CA, Goldstein ES, et al. Is perianal Crohn's disease associated with intestinal fistulization? Am J Gastroenterol 2005;100:1547-1549.

24. Ruemmele FM, Veres G, Kolho KL, et al. Consensus guidelines of ECCO/ESPGHAN on the medical management of pediatric Crohn's disease. J Crohns Colitis 2014;8:1179-1207.

25. Tarrant KM, Barclay ML, Frampton CM, Gearry RB. Perianal disease predicts changes in Crohn's disease phenotyperesults of a population-based study of inflammatory bowel disease phenotype. Am J Gastroenterol 2008;103:3082-3093.

26. Yoon JY, Cheon JH, Park SJ, Kim TI, Kim WH. Effects of perianal involvement on clinical outcomes in Crohn's disease over 10 years. Gut Liver 2018;12:297-305.

27. Kaur M, Panikkath D, Yan X, et al. Perianal Crohn's disease is associated with distal colonic disease, stricturing disease behavior, IBD-associated serologies and genetic variation in the JAK-STAT pathway. Inflamm Bowel Dis 2016;22:862869.

28. Lee YJ, Kim KM, Jang JY, Song K. Association of TNFSF15 polymorphisms in Korean children with Crohn's disease. Pediatr Int 2015;57:1149-1153.

29. Van Assche G, Vermeire S, Rutgeerts P. The potential for disease modification in Crohn's disease. Nat Rev Gastroenterol Hepatol 2010;7:79-85.

30. Kang B, Choi SY, Kim HS, Kim K, Lee YM, Choe YH. Mu- cosal healing in paediatric patients with moderate-to-severe luminal Crohn's disease under combined immunosuppression: escalation versus early treatment. J Crohns Colitis 2016;10:1279-1286.

31. Nuti F, Civitelli F, Bloise S, et al. Prospective evaluation of the achievement of mucosal healing with anti-TNF- $\alpha$ therapy in a paediatric Crohn's disease cohort. J Crohns Colitis 2016;10:5-12.

32. Reinink AR, Lee TC, Higgins PD. Endoscopic mucosal healing predicts favorable clinical outcomes in inflammatory bowel disease: a meta-analysis. Inflamm Bowel Dis 2016;22:1859-1869.

33. Ordás I, Feagan BG, Sandborn WJ. Early use of immunosuppressives or TNF antagonists for the treatment of Crohn's disease: time for a change. Gut 2011;60:1754-1763.

34. Walters TD, Kim MO, Denson LA, et al. Increased effectiveness of early therapy with anti-tumor necrosis factor- $a$ vs an immunomodulator in children with Crohn's disease. Gastroenterology 2014;146:383-391.

35. Cosnes J, Nion-Larmurier I, Beaugerie L, Afchain P, Tiret E, Gendre JP. Impact of the increasing use of immunosuppressants in Crohn's disease on the need for intestinal surgery. Gut 2005;54:237-241.

36. Camus M, Seksik P, Bourrier A, et al. Long-term outcome of patients with Crohn's disease who respond to azathioprine. Clin Gastroenterol Hepatol 2013;11:389-394. 